\title{
Degenerately Doped Transition Metal Dichalcogenides as Ohmic Homojunction Contacts to Transition Metal Dichalcogenide Semiconductors
}

\author{
Zhibin Gao, ${ }^{\dagger \ddagger}$ Zhixian Zhou, and David Tománek*,†,§ \\ $\dagger$ Physics and Astronomy Department, Michigan State University, East Lansing, Michigan \\ 48824, USA \\ $\ddagger$ Center for Phononics and Thermal Energy Science, School of Physics Science and \\ Engineering, Tongji University, 200092 Shanghai, P. R. China \\ ฯPhysics and Astronomy Department, Wayne State University, Detroit, Michigan 48201, \\ USA \\ $\S$ Mandelstam Institute for Theoretical Physics and School of Physics, University of the \\ Witwatersrand, 2050 Johannesburg, South Africa \\ E-mail: tomanek@pa.msu.edu
}

\begin{abstract}
In search of an improved strategy to form lowresistance contacts to $\mathrm{MoS}_{2}$ and related semiconducting transition metal dichalcogenides, we use $a b$ initio density functional electronic structure calculations in order to determine the equilibrium geometry and electronic structure of $\mathrm{MoO}_{3} / \mathrm{MoS}_{2}$ and $\mathrm{MoO}_{2} / \mathrm{MoS}_{2}$ bilayers. Our results indicate that, besides a rigid band shift associated with charge transfer, the presence of molybdenum oxide modifies the electronic structure of $\mathrm{MoS}_{2}$ very little. We find that the charge transfer in the bilayer provides a sufficient degree of hole doping to $\mathrm{MoS}_{2}$, resulting in a highly transparent contact region.
\end{abstract}

\section{Keywords}

transition metal dichalcogenides, contacts, $a b$ initio calculations, electronic structure, charge transfer, doping, band offset

One of the key challenges in 2D semicon- ductor physics is finding better ways to inject charge carriers through transparent, ohmic con$\operatorname{tacts}^{13}$ in order to reduce power dissipation and increase carrier mobility. ${ }^{45}$ This problem does not occur in 3D semiconductors, where degenerate doping, resulting in transparent contacts to metal electrodes, is achieved by ion implantation. ${ }^{6}$ That approach is not practical in atomically thin 2D layers, since bombardment by ions with a finite penetration depth would not only implant, but also knock out atoms from the channel. ${ }^{7}$ Several alternative approaches have been explored in the past. The most common among these is doping by elemental substitution $[-10$ and by surface charge transfer. ${ }^{7 / 11 \mid 12}$ Doping the entire structure does improve charge injection in the contact region, but also turns the channel metallic, reducing its switching capability. ${ }^{13}$ Significant local doping by surface charge transfer may also be achieved using adsorbed molecules, but typically suffers from lack of chemical and thermal stability. Substitutional doping yields a stable structure, but cannot be strictly limited to the contact regions only. It generally causes structural 
changes and introduces scattering centers in the 2D channel, thus degrading its transport properties. Direct local contact between a 2D semiconductor and a metal with a favorable work function appears attractive, but typically results in a strong hybridization at the interface, causing the formation of mid-gap states and Fermi level pinning. $\frac{314}{17}$ One way to reduce these negative side effects of a metal contact is to insert an ultra-thin insulating layer such as h-BN or $\mathrm{MgO}$ in-between the semiconducting channel and the metal. $\frac{[18] 20}{2}$ This approach favorably modifies the work function of the metal while reducing the net charge transfer between the contact metal and the channel, suppresses the formation of interlayer gap states and Fermi level pinning. The drawback of this approach is widening the vertical tunnel barrier between the contact metal and the channel. Whereas formation of mid-gap states can be avoided when using substitutionally heavily doped or metallic 2D transition metal dichalcogenides in van der Waals contact with the channel, 10121 the vertical tunnel barrier can not be eliminated. An unusual way to optimize contacts to $2 \mathrm{D}$ semiconductors, which does not involve charge transfer to the channel, is by phase engineering. $22+24$ In this approach, an in-layer homo-junction is formed when locally converting the semiconducting $2 \mathrm{H}$ phase of the channel material such as $\mathrm{MoS}_{2}$ to to a metastable, metallic $1 \mathrm{~T}$ phase. This approach is, however, restricted to selected materials and the phase stability is limited. All of the above strategies have significant drawbacks, which make continuing search for suitable alternatives highly desirable.

We propose a previously unexplored approach to achieve degenerate hole doping in a local region of a semiconducting $\mathrm{MoS}_{2}$ channel that is placed in direct contact with electronegative materials such as $\mathrm{MoO}_{3}$ or $\mathrm{MoO}_{2}$ using van der Waals assembly. To prove the viability of this approach, we perform ab initio density functional calculations of the vertical heterostructure using density functional theory (DFT), which is known to describe charge distribution and doping correctly. We find that the charge transfer between the doping layer and the channel is significant and results in de-
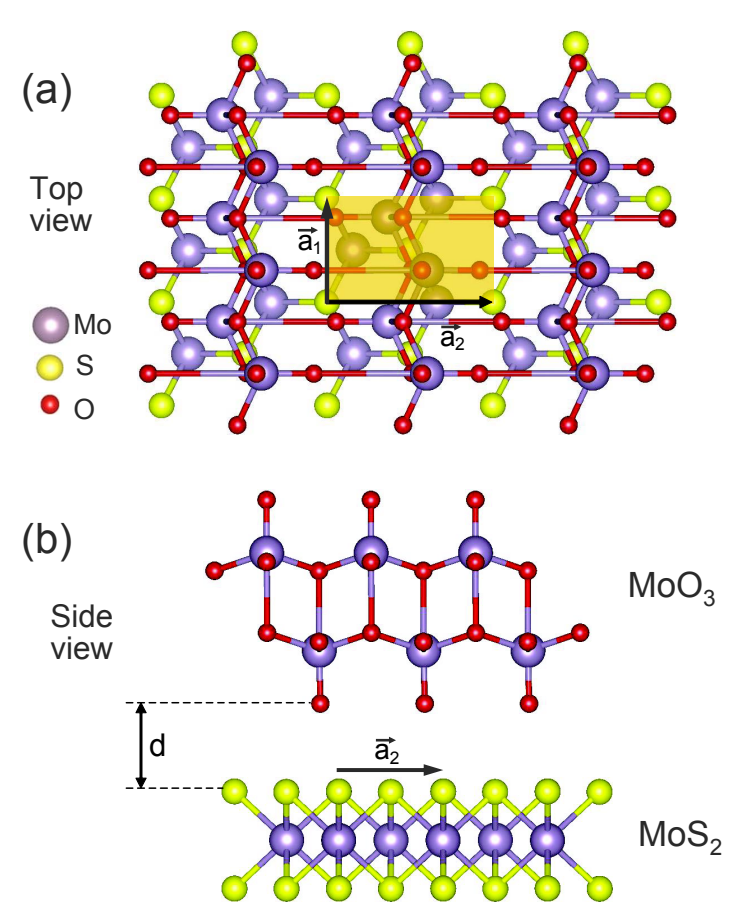

Figure 1: Ball-and-stick model of the $\mathrm{MoO}_{3} / \mathrm{MoS}_{2}$ bilayer in (a) top view and (b) side view. The unit cell is indicated by yellow shading in (a). $\quad \vec{a}_{1}$ and $\vec{a}_{2}$ are the lattice vectors spanning the $2 \mathrm{D}$ lattice and $d$ is the inter-layer distance.

generate doping of the $\mathrm{MoS}_{2}$ channel by the adjacent molybdenum oxide layer. With only negligible hybridization at the bilayer interface and its negative side effects, the dominant electronic structure change is a net charge transfer giving rise to an interface dipole, which causes a rigid shift ${ }^{21}$ of the $\mathrm{MoS}_{2}$ bands in the contact region and turns it metallic. In this case, no vertical tunnel barrier will form when the heavily doped and highly conducting contact region of the channel material is contacted by a metal on the side opposite to molybdenum oxide.

In common Si-based 3D semiconductor devices, charge transport is dominated by the intrinsic properties of the channel. Contacts do not play a significant role, since the contact region is degenerately doped by ion implantation, which allows for barrier-free charge injection from a metal electrode. ${ }^{6}$ The device characteristics change significantly in low-dimensional semiconductors, where contacts play the dominant role due to significant Schottky barriers and an associated wide depletion region caused 
by insufficient screening. The key role of Schottky barriers in the contact region has been recognized early in $1 \mathrm{D}$ semiconducting carbon nanotubes with metal contacts, ${ }^{25}$ with similar behavior extending to $2 \mathrm{D}$ semiconductors with metal contacts. 26 There, the contact resistance can be reduced, albeit not eliminated, by selecting contact metals with a work function aligned with the valence band maximum (VBM) or conduction band minimum (CBM), depending on the type of carriers to be injected. 1427

Our alternative approach is to to create a homo-junction between a doped and a pristine segment of a $2 \mathrm{D}$ semiconductor such as $\mathrm{MoS}_{2}$. Key to this approach is the use of van der Waals assembly to contact the channel by a highly electronegative material that locally provides degenerate hole doping. The material of choice in this study is $\mathrm{MoO}_{3}$ with a very high work function $\underline{28}$ of $6.7 \mathrm{eV}$. This material has emerged as a promising surface acceptor material for a wide variety of systems including diamond thin films, graphene, and transition metal dichalcogenides (TMDs). ${ }^{29} \mathrm{Be}$ cause the CBM of $\mathrm{MoO}_{3}$ lies below the VBM of most semiconductors including TMDs, molybdenum oxide is a viable candidate to provide degenerate doping to $2 \mathrm{D}$ semiconductors.

\section{Results}

\section{$\mathrm{MoS}_{2}$ in contact with $\mathrm{MoO}_{3}$}

The structure of isolated $\mathrm{MoO}_{3}$ and $\mathrm{MoS}_{2}$ monolayers, as well as that of the bilayer, is shown in Figure 1. The Bravais lattice of $\mathrm{MoO}_{3}$ has a rectangular unit cell containing 2 Mo and $6 \mathrm{O}$ atoms with $a_{1}=3.71 \AA$ and $a_{2}=3.95 \AA$. The calculated cohesive energy per formula unit of a free-standing $\mathrm{MoO}_{3}$ monolayer is $E_{c o h}=24.62 \mathrm{eV}$ with respect to isolated atoms.

$\mathrm{MoS}_{2}$ forms a triangular lattice with the lattice constant $a=3.18 \AA$. The corresponding lattice constants of a conventional rectangular unit cell containing 2 Mo and $4 \mathrm{~S}$ atoms, useful when discussing epitaxy with $\mathrm{MoO}_{3}$, are $a_{1}=3.18 \AA$ and $a_{2}=5.51 \AA$. The calculated co- hesive energy per formula unit of free-standing $\mathrm{MoS}_{2}$ is $E_{\text {coh }}=16.37 \mathrm{eV}$ with respect to isolated atoms.

Since the computational approach to investigate infinite structures requires a periodic array of identical unit cells and since the $\mathrm{MoO}_{3}$ and the $\mathrm{MoS}_{2}$ layers are incommensurate, we need to enforce commensurability by subjecting the individual layers to in-layer stain at some energy cost. To obtain quantitative understanding of the effect of strain on our results, we distinguished the smaller supercell A containing $4 \mathrm{Mo}, 6 \mathrm{O}$, and $4 \mathrm{~S}$ atoms, shown in Figure 1(a), from the larger supercell $B$ that contains $44 \mathrm{Mo}, 72 \mathrm{O}$, and $40 \mathrm{~S}$ atoms. Both supercells are shown in the Supporting Information. The primary reason to consider the smaller supercell A, which is highly strained, is to obtain an intuitive understanding of the Physics. The larger supercell B is less strained and expected to provide a better quantitative agreement with the real system.

The strain energy in stretched and compressed $\mathrm{MoO}_{3}$ and $\mathrm{MoS}_{2}$ monolayers as well as in the bilayer is shown in Figure 2, The deformation energies $\Delta E$, shown as a function of the lattice constants in Figure $2(\mathrm{a})-2(\mathrm{e})$, allow to judge the in-plane compressibility of the $\mathrm{MoO}_{3} / \mathrm{MoS}_{2}$ bilayer and its components. The inter-layer interaction energy in the bilayer system with supercell A, shown in Figure 2(f), has been fitted by the Morse potential $\Delta E=E_{0}\left[1-\exp \left(\xi\left[d-d_{0}\right]\right)\right]$.

We find results based on DFT-PBE to be in very good agreement with those based on the DFT-optB86b-vdW functional. This indicates that the role of van der Waals interactions, which are specifically addressed in the latter functional, is only secondary. Since our results for both supercells indicate a significant charge redistribution within the vertical heterojunction, the role of the Coulomb attraction between adjacent $\mathrm{MoO}_{3}$ and $\mathrm{MoS}_{2}$ layers is more significant. We note that Coulomb interaction is described adequately by the DFT formalism.

The relaxed geometry of the the bilayer structure with the rectangular supercell $\mathrm{A}$ is characterized by the lattice constants $a_{1}=3.34 \AA$, $a_{2}=5.42 \AA$, and the unit cell area $A_{A}=$ 

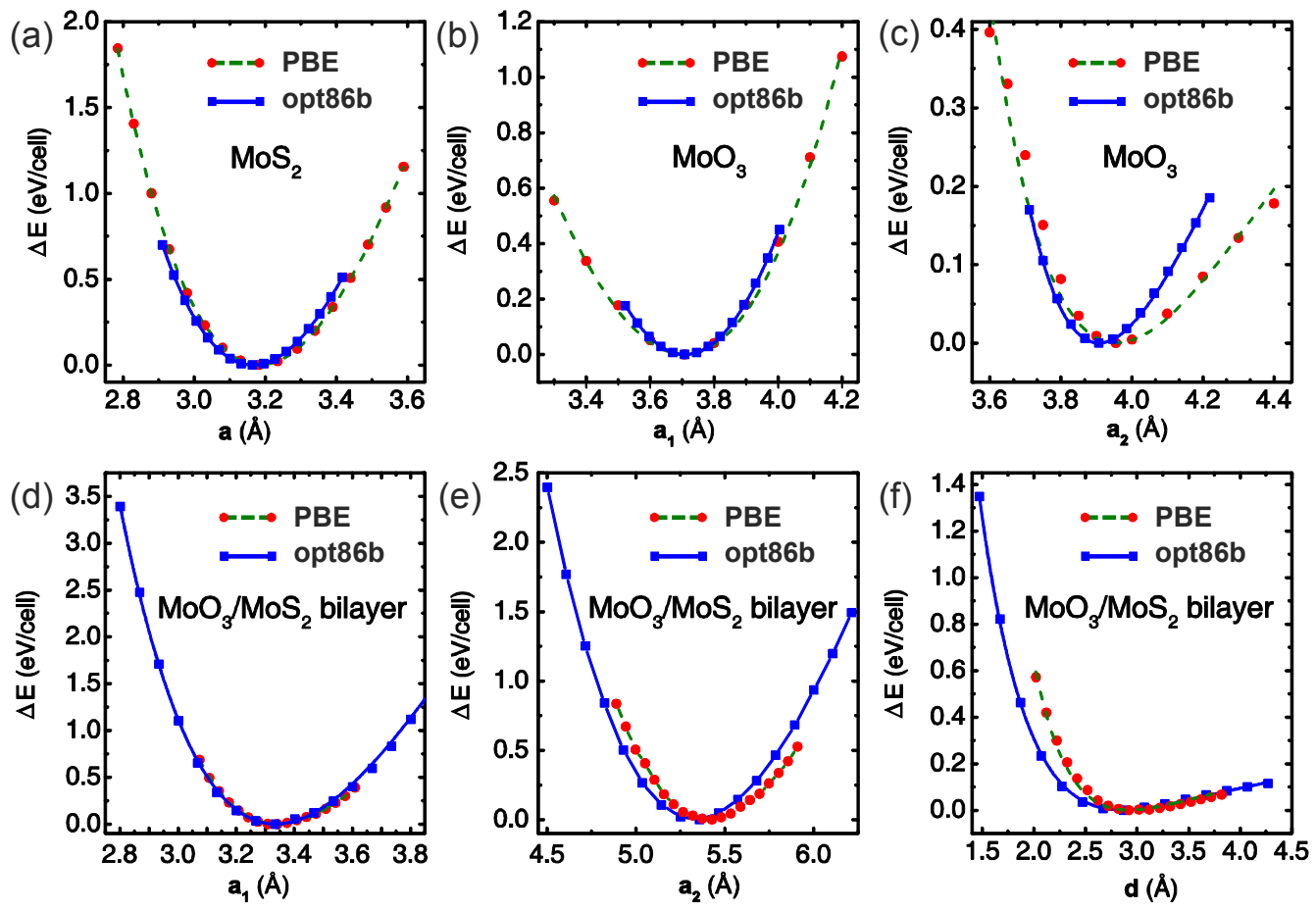

Figure 2: Energy change $\Delta E$ per unit cell as a function of the lattice parameters. Monolayer results are presented for (a) $\mathrm{MoS}_{2}$ with threefold symmetry and lattice constant $a$ and (b),(c) $\mathrm{MoO}_{3}$ with lattice constants $a_{1}$ and $a_{2}$. The unit cells we consider contain 3 atoms in (a) and 8 atoms in (b),(c). $\mathrm{MoO}_{3} / \mathrm{MoS}_{2}$ bilayer results representing 14-atom unit cells show $\Delta E$ as a function of (d) $a_{1}$, (e) $a_{2}$, and (f) the interlayer distance $d$. The dashed lines in (a-d) and (f) are fits by the Morse potential. The dashed line in (e) is a guide to the eye. The legend in the panels specifies the symbols and line types for results based on DFT-PBE and also the DFT-optB86b-vdW functional, which specifically considers the van der Waals interaction.

$18.11 \AA^{2}$. We note that the optimum values of $a_{1}$ and $a_{2}$ in the bilayer lie in-between the values of the isolated monolayers. In the $\mathrm{MoO}_{3}$ layer within the relaxed bilayer with supercell A, $a_{1}$ is compressed by $9.8 \%$ and $a_{2}$ is stretched by $37.0 \%$ at the energy cost of $5.5 \mathrm{eV} / \mathrm{nm}^{2}$. In the $\mathrm{MoS}_{2}$ layer in the same geometry, $a_{1}$ is stretched by $5.1 \%$ and $a_{2}$ is compressed by $1.6 \%$ at the energy cost of $0.9 \mathrm{eV} / \mathrm{nm}^{2}$. The attractive interaction energy between the strained layers amounts to $0.1 \mathrm{eV} / \mathrm{nm}^{2}$.

The relaxed geometry of the the bilayer structure with the larger rectangular supercell B is characterized by the lattice constants $a_{1}=$ $15.87 \AA$ and $a_{2}=11.07 \AA$ and the unit cell area $A_{B}=175.72 \AA^{2}$. We note again that the optimum $a_{1}$ and $a_{2}$ values in the bilayer are inbetween the values of the isolated monolayers. The relative deformations and the deformation energies are much smaller in the larger supercell
B. In the $\mathrm{MoO}_{3}$ layer within the relaxed bilayer with supercell B, $a_{1}$ is stretched by $7.0 \%$ and $a_{2}$ is compressed by $6.7 \%$ at the energy cost of $0.3 \mathrm{eV} / \mathrm{nm}^{2}$. In the $\mathrm{MoS}_{2}$ layer within the same relaxed bilayer geometry, $a_{1}$ is compressed by $0.2 \%$ and $a_{2}$ is stretched by $0.5 \%$ at the energy cost of $0.6 \mathrm{eV} / \mathrm{nm}^{2}$. The attractive interaction energy between the strained layers in the bilayer amounts to $0.7 \mathrm{eV} / \mathrm{nm}^{2}$, nearly compensating for the deformation energy of the individual layers.

To obtain a basic understanding of what is happening in the system electronically, we first consider the smaller supercell A of the $\mathrm{MoO}_{3} / \mathrm{MoS}_{2}$ bilayer. The electronic band structure based on DFT-PBE Kohn-Sham eigenvalues, shown in Figure 3(a), suggests that the system is semimetallic. A superposition of electronic band structures of isolated $\mathrm{MoO}_{3}$ and $\mathrm{MoS}_{2}$ monolayers, constrained to their re- 

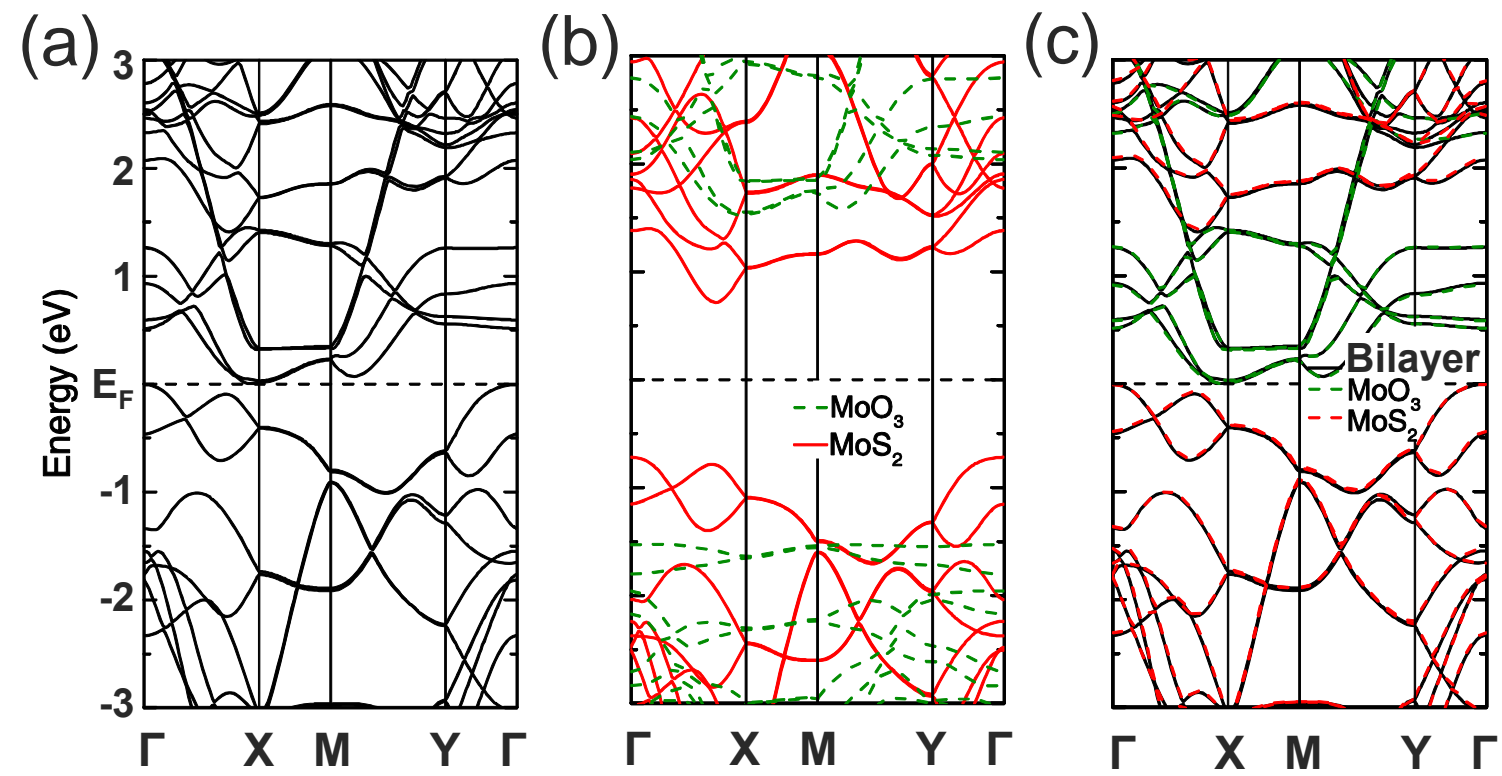

Figure 3: (a) Electronic band structure of a $\mathrm{MoO}_{3} / \mathrm{MoS}_{2}$ bilayer with supercell A. (b) Superposition of the electronic band structure of isolated monolayers of $\mathrm{MoS}_{2}$ (solid red lines) and $\mathrm{MoO}_{3}$ (dashed green lines). (c) Superposition of the $\mathrm{MoS}_{2}$ bands in panel (b), shifted rigidly up by $0.711 \mathrm{eV}$, and the $\mathrm{MoO}_{3}$ bands, shifted rigidly down by $1.523 \mathrm{eV}$. The combined band structure in (c) is superposed to that of the $\mathrm{MoO}_{3} / \mathrm{MoS}_{2}$ bilayer in panel (a).
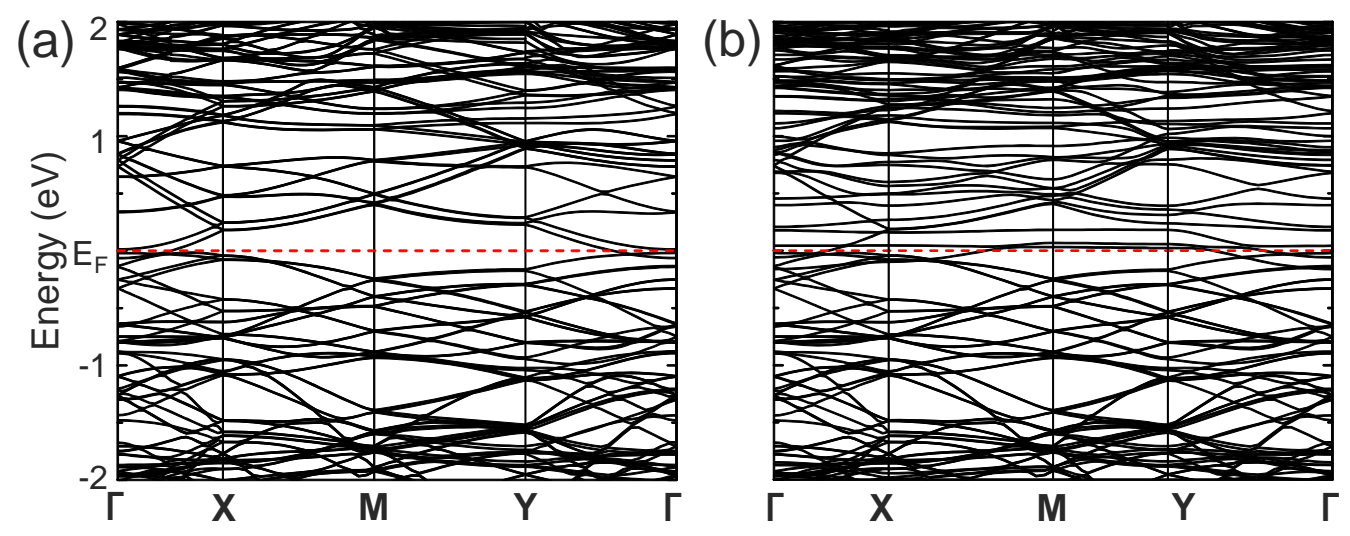

Figure 4: Electronic band structure of an $\mathrm{MoO}_{3-x} / \mathrm{MoS}_{2}$ bilayer based on DFT-PBE and represented by supercell B containing 156 atomic sites in total. Results for a defect-free system $(x=0)$ (a) are compared to those for a defective system $(x>0)$ with one oxygen vacancy in each unit cell (b). The Fermi level is indicated by the red dashed line.

spective structure in the bilayer, is shown in Figure 3(b), with the Fermi levels aligned at mid-gap. In view of the fact that fundamental gaps are typically underestimated in the KohnSham DFT-PBE spectrum, we expect that the $\mathrm{MoO}_{3} / \mathrm{MoS}_{2}$ bilayer with supercell A, as well as the $\mathrm{MoO}_{3}$ and $\mathrm{MoS}_{2}$ monolayers, should all be semiconducting. This expectation is confirmed in the corresponding band structure and density of states obtained using the hybrid DFT-
HSE06 exchange-correlation functional, which is presented in the Supporting Information. Kohn-Sham spectra based on DFT-HSE06 typically have wider fundamental band gaps that agree better with observed electronic spectra than spectra based on DFT-PBE.

To inspect the level of hybridization at the interface, we superposed the DFT-PBE band structure of the bilayer with the bands of an isolated $\mathrm{MoS}_{2}$ monolayer, shifted rigidly up 

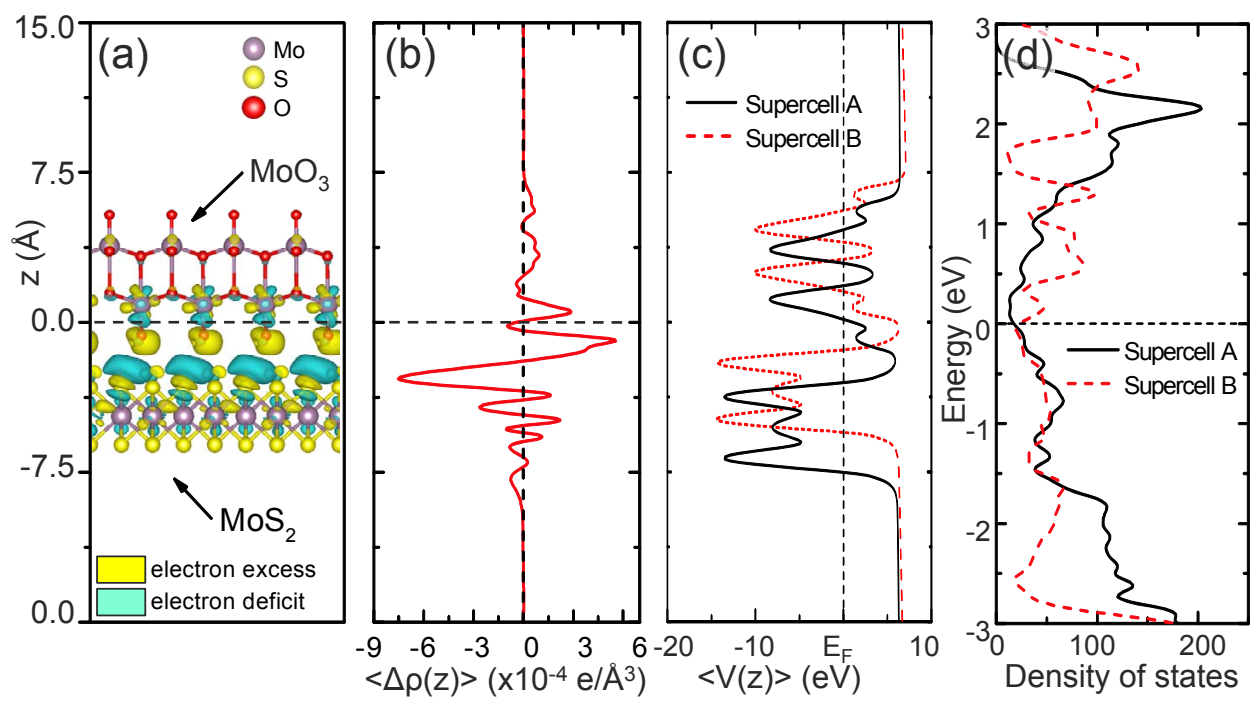

Figure 5: Electronic structure changes associated with assembling the $\mathrm{MoO}_{3} / \mathrm{MoS}_{2}$ bilayer from isolated monolayers. (a) Charge density difference $\Delta \rho=\rho\left(\mathrm{MoO}_{3} / \mathrm{MoS}_{2}\right)-\rho\left(\mathrm{MoO}_{3}\right)-\rho\left(\mathrm{MoS}_{2}\right)$. $\Delta \rho$ is shown by isosurfaces bounding regions of electron excess at $+1.0 \times 10^{-3} \mathrm{e} / \AA^{3}$ (yellow) and electron deficiency at $-1.0 \times 10^{-3} \mathrm{e} / \AA^{3}$ (blue). (b) $\langle\Delta \rho(z)\rangle$ averaged across the $x-y$ plane of the layers. The black solid reference line is a guide to the eye. $z$ indicates the position of the plane. (c) Electrostatic potential $\langle V(z)\rangle$ in the bilayer, averaged across the $\mathrm{x}-\mathrm{y}$ plane, with $z$ denoting the position of the plane. (d) Density of states (DOS) of the bilayer, convoluted by a Gaussian with a full-width at half-maximum of $0.1 \mathrm{eV}$. (c) and (d) display results for supercell A (solid black line) and supercell B (dashed red line), defined in the text.

by $0.711 \mathrm{eV}$, and an $\mathrm{MoO}_{3}$ monolayer, shifted rigidly down by $1.523 \mathrm{eV}$. Comparing the band superposition with the bilayer band structure in Figure 3(c), we find only a minimum degree of hybridization between the individual layers. The same small degree of hybridization also occurs in DFT-HSE06 calculations. Similar to DFT-PBE results, the DFT-HSE06 band structure of the bilayer is represented well by a superposition of $\mathrm{MoS}_{2}$ monolayer bands, shifted rigidly up by $0.660 \mathrm{eV}$, and $\mathrm{MoO}_{3}$ monolayer bands, shifted rigidly down by $2.461 \mathrm{eV}$.

In truth, the above semiconducting behavior is not intrinsic to the $\mathrm{MoO}_{3} / \mathrm{MoS}_{2}$ bilayer, but rather linked to using the small supercell A. DFT-PBE band structure results for the bilayer represented by the much larger and more adequate supercell B, presented in Fig. 4(a), indicate a much higher degree of metallicity at the Fermi level. In realistic $\mathrm{MoO}_{3-x}$ systems, there are non-stoichiometric composition variations with oxygen vacancies. $\frac{3133}{13}$ As shown in Fig. 4(b), such oxygen vacancies always cause partly filled bands associated with metallic be- havior.

The charge redistribution in the bilayer is shown in Figure 5. The net charge transferred from the $\mathrm{MoS}_{2}$ to the $\mathrm{MoO}_{3}$ layer is $\Delta \rho_{2 D}=$ $2.0 \times 10^{13} \mathrm{e} / \mathrm{cm}^{2}$ in the bilayer with the smaller supercell $\mathrm{A}$ and $\Delta \rho_{2 D}=4.5 \times 10^{13} \mathrm{e} / \mathrm{cm}^{2}$ in the bilayer with the larger supercell $\mathrm{B}$. When divided by the thickness $t \approx 0.4 \mathrm{~nm}$ of the $\mathrm{MoS}_{2}$ channel, the charge transfer density ranges from $\Delta \rho=5 \times 10^{20}-1 \times 10^{21} \mathrm{e} / \mathrm{cm}^{3}$. This is high enough and considered degenerate doping, since $E_{F}$ has been moved into the valence band. Having achieved degenerate doping in the contact region of the bilayer, the tunnel barrier to a metal contact at the side opposite to the doping layer should also be negligibly small and of no consequence.

\section{$\mathrm{MoS}_{2}$ in contact with $\mathrm{MoO}_{2}$}

As mentioned before, experimental studies report non-stoichiometric variations of $\mathrm{MoO}_{3-x}$ with oxygen vacancies and amorphous structure, $\stackrel{3133}{ }$ which cannot be modelled as periodic 
structures. As a step in the direction of molybdenum oxide with a lower oxygen concentration, we consider instead the $\mathrm{MoO}_{2}$ stoichiometry. $\mathrm{MoO}_{2}$ has been discussed theoretically and found to form a stable honeycomb lattice, $\stackrel{34}{\text { dif- }}$ ferent from the $\mathrm{MoO}_{3}$ lattice with a rectangular unit cell. Since $\mathrm{MoO}_{2}$ shares the same sixfold symmetry with $\mathrm{MoS}_{2}$, we have considered this system as a potential alternative to $\mathrm{MoO}_{3}$ in hole-doping $\mathrm{MoS}_{2}$.

(a)

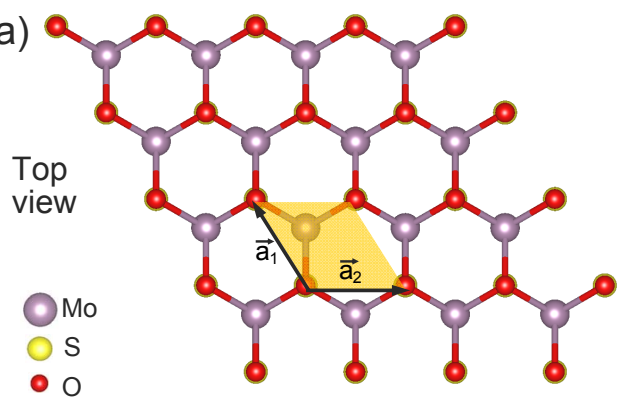

(b)

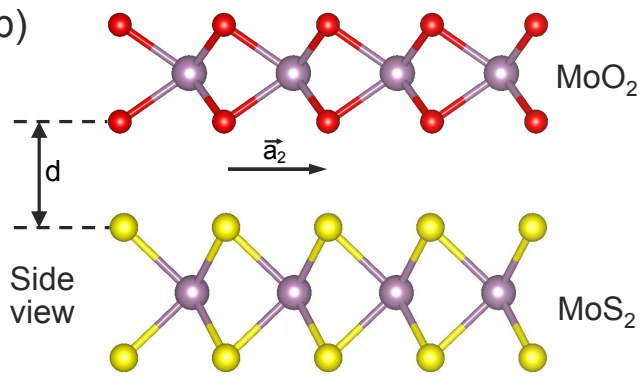

Figure 6: Ball-and-stick model of the $\mathrm{MoO}_{2} / \mathrm{MoS}_{2}$ bilayer in (a) top view and (b) side view. The unit cell is indicated by yellow shading in (a). $\quad \vec{a}_{1}$ and $\vec{a}_{2}$ are the lattice vectors spanning the 2D lattice and $d$ is the inter-layer distance.

The structure of the isolated $\mathrm{MoO}_{2}$ and $\mathrm{MoS}_{2}$ monolayers, as well as the bilayer structure, are shown in Figure 6. Unlike $\mathrm{MoO}_{3}, \mathrm{MoO}_{2}$ forms a honeycomb lattice with the lattice constant $a=2.83 \AA$. The calculated cohesive energy per formula unit of a free-standing $\mathrm{MoO}_{2}$ monolayer is $E_{c o h}=19.65 \mathrm{eV}$ with respect to isolated atoms.

We describe the $\mathrm{MoO}_{2} / \mathrm{MoS}_{2}$ bilayer by an epitaxial triangular Bravais lattice with a basis and one formula unit per unit cell. The optimum lattice constant is $a=\left|\overrightarrow{a_{1}}\right|=\left|\overrightarrow{a_{2}}\right|=2.99 \AA$ and the unit cell area is $A=7.72 \AA^{2}$. We note that the optimum value of $a$ in the bilayer are in-between the values of the isolated monolayers. In the $\mathrm{MoO}_{2}$ layer, $a$ is stretched by $5.5 \%$ at the energy cost of $5.2 \mathrm{eV} / \mathrm{nm}^{2}$. In the $\mathrm{MoS}_{2}$ layer, $a$ is compressed by $6.1 \%$ at the energy cost of $4.5 \mathrm{eV} / \mathrm{nm}^{2}$. The attractive interaction energy between the strained layers amounts to $0.1 \mathrm{eV} / \mathrm{nm}^{2}$.

Similar to $\mathrm{MoO}_{3}$ interacting with $\mathrm{MoS}_{2}$, also interacting monolayers of $\mathrm{MoO}_{2}$ and $\mathrm{MoS}_{2}$ need to be strained to form a commensurate structure. The unit cell of the bilayer, shown in Figure 6(b), contains $2 \mathrm{Mo}, 2 \mathrm{~S}$, and $2 \mathrm{O}$ atoms. The strain energy in stretched and compressed $\mathrm{MoO}_{2}$ and $\mathrm{MoS}_{2}$ monolayers as well as the bilayer is shown in Figure 7. Also in this system, we find results based on DFT-PBE to be in very good agreement with those based on the DFToptB86b-vdW functional. This indicates that van der Waals interactions, which are specifically considered in the latter functional, play only a secondary role.

The electronic band structure of the $\mathrm{MoO}_{2} / \mathrm{MoS}_{2}$ bilayer, shown in Figure 8(a), indicates that the system is metallic as hoped for. A superposition of electronic band structures of isolated $\mathrm{MoO}_{2}$ and $\mathrm{MoS}_{2}$ monolayers, constrained to their respective structure in the bilayer, is shown in Figure 8(b), with the Fermi levels of the individual layers aligned. In contrast to the $\mathrm{MoS}_{2}$ channel, our results suggest that $\mathrm{MoO}_{2}$ should be metallic. In view of the fact that DFT-PBE calculations typically underestimate band gaps, we realistically expect $\mathrm{MoO}_{2}$ to be a semiconductor with a narrow, indirect band gap instead. This is confirmed by DFT-HSE06 band structure and density of states results, presented in the Supporting Information, which indicate a $0.5 \mathrm{eV}$-wide indirect band gap in an isolated $\mathrm{MoO}_{2}$ monolayer. To inspect the level of hybridization at the $\mathrm{MoO}_{2} / \mathrm{MoS}_{2}$ interface, we superposed the band structure of the bilayer with the bands of the $\mathrm{MoS}_{2}$ monolayer, shifted rigidly up by $0.768 \mathrm{eV}$, and the unshifted bands of the $\mathrm{MoO}_{2}$ monolayer. Comparing the band superposition with the bilayer band structure in Figure 8(c), we find only a minimum degree of hybridization in the system. The same low degree of hybridization, but a significant charge trans- 

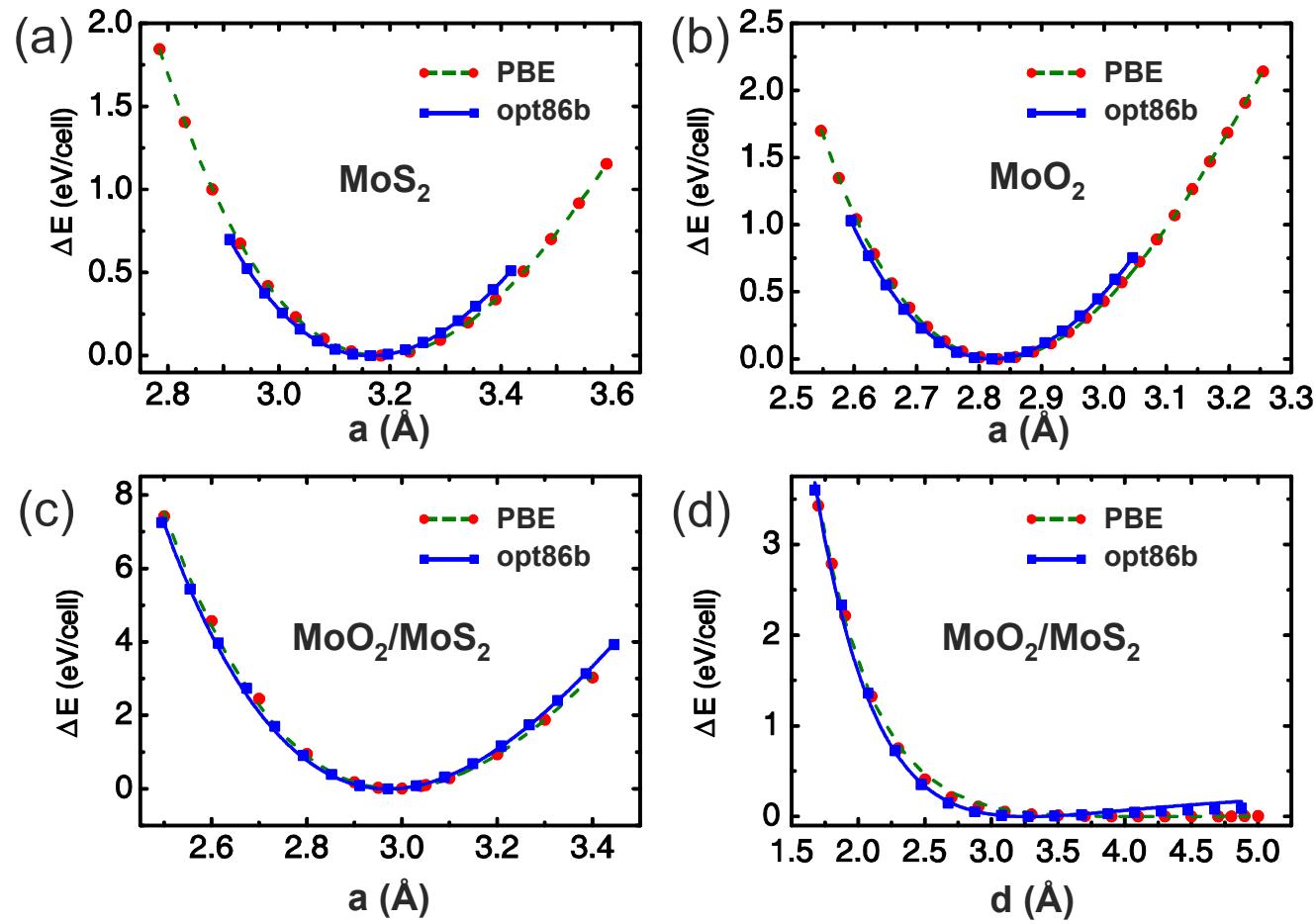

Figure 7: Energy change $\Delta E$ per unit cell as a function of the lattice constant $a$ in monolayers of (a) $\mathrm{MoS}_{2}$ and (b) $\mathrm{MoO}_{2}$. $\Delta \mathrm{E}$ per unit cell in $\mathrm{MoO}_{2} / \mathrm{MoS}_{2}$ bilayers as a function of (c) the lattice constant $a$ and (f) the interlayer distance $d$. The dashed lines are fits by the Morse potential. The legend in the panels specifies the symbols and line types for results based on DFT-PBE and for those based on the DFT-optB86b-vdW functional, which specifically considers the van der Waals interaction.
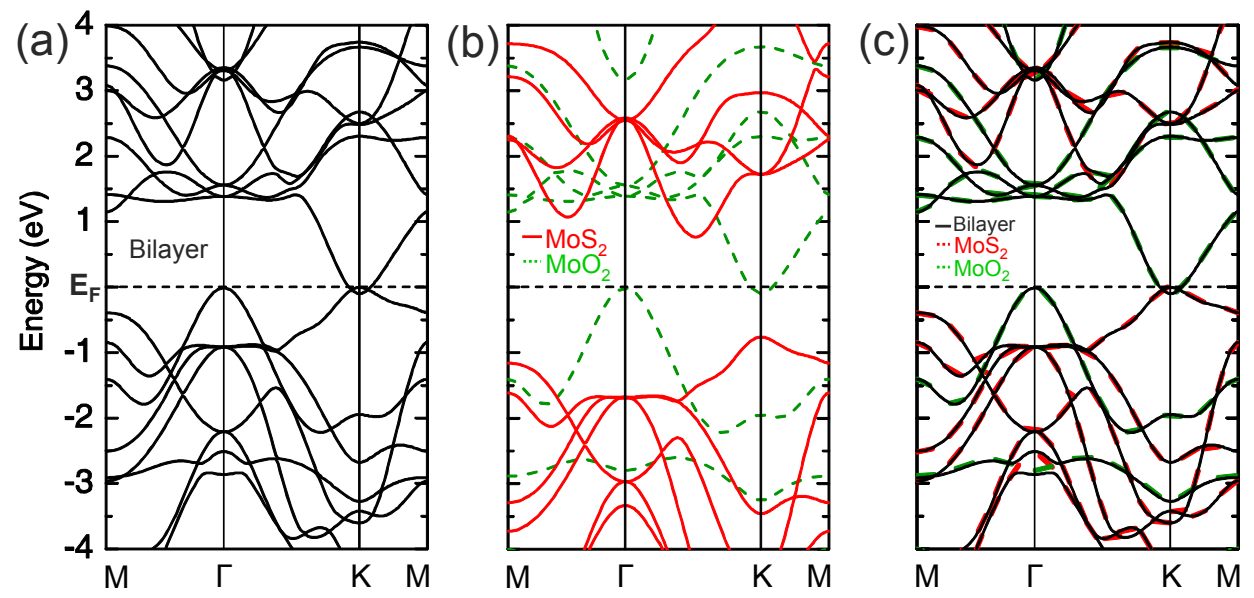

Figure 8: (a) Electronic band structure of a $\mathrm{MoO}_{2} / \mathrm{MoS}_{2}$ bilayer. (b) Superposition of the electronic band structure of isolated monolayers of $\mathrm{MoS}_{2}$ (solid red lines) and $\mathrm{MoO}_{2}$ (dashed green lines). (c) Superposition of the $\mathrm{MoS}_{2}$ bands in panel (b), shifted rigidly up by $0.768 \mathrm{eV}$, and the unshifted $\mathrm{MoO}_{2}$ bands. The combined band structure in (c) is superposed to that of the $\mathrm{MoO}_{2} / \mathrm{MoS}_{2}$ bilayer in panel (a).

fer from $\mathrm{MoS}_{2}$ to $\mathrm{MoO}_{2}$ causing a net metallic behavior of the bilayer, are also found in corresponding DFT-HSE06 band structure results presented in the Supporting Information.
The charge redistribution in the bilayer is shown in Figure 9. The net charge transferred from the $\mathrm{MoS}_{2}$ to the $\mathrm{MoO}_{2}$ layer is $\Delta \rho_{2 D}=1.7 \times 10^{13} \mathrm{e} / \mathrm{cm}^{2}$ in the bilayer. When 

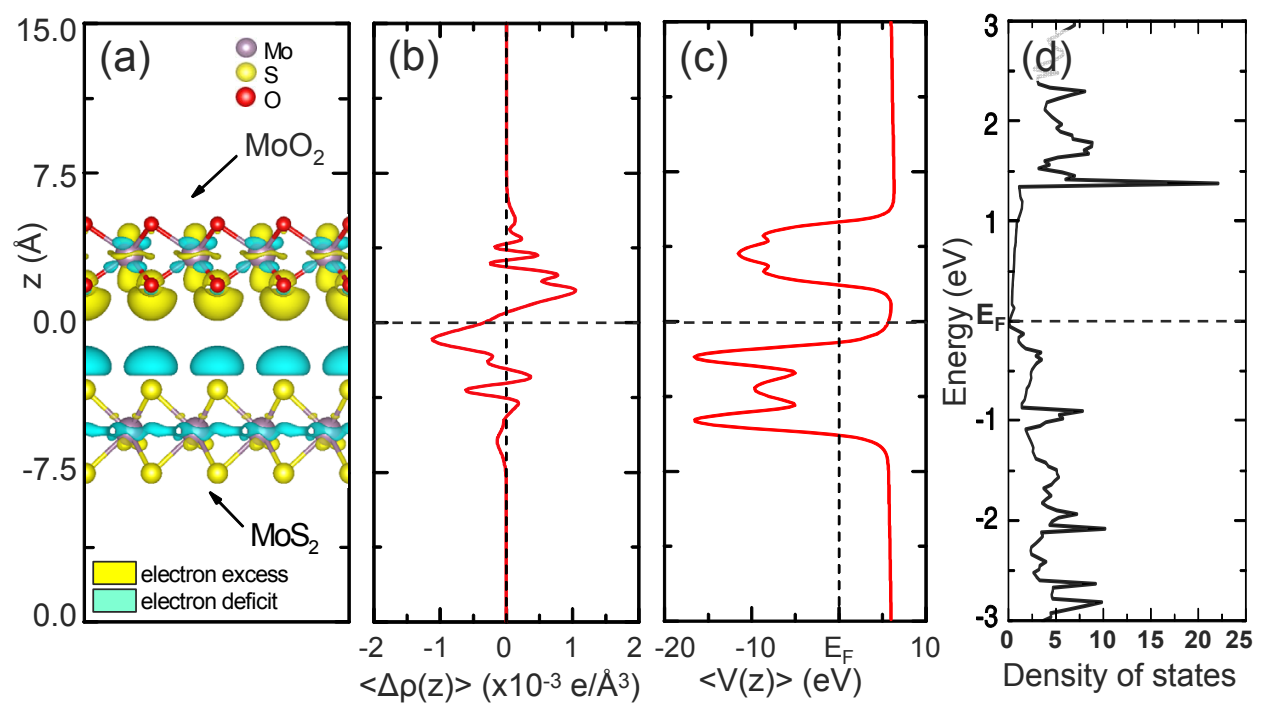

Figure 9: Electronic structure changes associated with assembling the $\mathrm{MoO}_{2} / \mathrm{MoS}_{2}$ bilayer from isolated monolayers. (a) Charge density difference $\Delta \rho=\rho\left(\mathrm{MoO}_{2} / \mathrm{MoS}_{2}\right)-\rho\left(\mathrm{MoO}_{2}\right)-\rho\left(\mathrm{MoS}_{2}\right)$. $\Delta \rho$ is shown by isosurfaces bounding regions of electron excess at $+1.0 \times 10^{-3} \mathrm{e} / \AA^{3}$ (yellow) and electron deficiency at $-1.0 \times 10^{-3} \mathrm{e} / \AA^{3}$ (blue). (b) $\langle\Delta \rho(z)\rangle$ averaged across the $x-y$ plane of the layers. The black solid reference line is a guide to the eye and $z$ indicates the position of the plane. (c) Electrostatic potential $\langle V(z)\rangle$ in the bilayer, averaged across the $\mathrm{x}-\mathrm{y}$ plane, with $z$ denoting the position of the plane. (d) Density of states (DOS) of the bilayer, convoluted by a Gaussian with a full-width at half-maximum of $0.1 \mathrm{eV}$.

divided by the thickness $t \approx 0.4 \mathrm{~nm}$ of the $\mathrm{MoS}_{2}$ channel, the charge transfer density amounts to $\Delta \rho=4 \times 10^{20} \mathrm{e} / \mathrm{cm}^{3}$, slightly lower than in the $\mathrm{MoO}_{3} / \mathrm{MoS}_{2}$ bilayer. This doping level is still considered to be degenerate, since $E_{F}$ has been moved into the valence band of the channel. With degenerate doping in the contact region of the bilayer, the tunnel barrier to a metal contact on the side opposite to the doping layer should again be negligibly small and of no consequence.

\section{Discussion}

As expected in the outset, our numerical results based on a Bader charge analysis indicate a significant charge transfer between adjacent $\mathrm{MoO}_{3-x}$ and $\mathrm{MoS}_{2}$ layers in the bilayer geometry. To validate the amount of charge transferred between the layers, we compared the charge density difference $\Delta \rho_{2 D}$ based on the current DFT-PBE nonlocal exchange correlation functional to those based on the local DFT-LDA functional. The specific values for $\Delta \rho_{2 D}$ in $\mathrm{MoO}_{3} / \mathrm{MoS}_{2}$ are $5.55 \times 10^{13} \mathrm{e} / \mathrm{cm}^{2}$ (LDA for supercell B), $4.49 \times 10^{13} \mathrm{e} / \mathrm{cm}^{2}(\mathrm{PBE}$ for supercell B), $2.09 \times 10^{13} \mathrm{e} / \mathrm{cm}^{2}$ (LDA for supercell A), $1.97 \times 10^{13} \mathrm{e} / \mathrm{cm}^{2}$ (PBE for supercell A). The corresponding values for $\Delta \rho_{2 D}$ in $\mathrm{MoO}_{2} / \mathrm{MoS}_{2}$ are $1.80 \times 10^{13} \mathrm{e} / \mathrm{cm}^{2}(\mathrm{LDA})$ and $1.67 \times 10^{13} \mathrm{e} / \mathrm{cm}^{2}(\mathrm{PBE})$. These results clearly indicate that the effect of the exchangecorrelation functional on the charge transfer is very small. Since the treatment of exchange and correlation in the hybrid DFT-HSE06 functional with a slowly decaying Fock exchange is fundamentally different from the more localized form form in DFT-LDA and DFT-PBE functionals, $\frac{35136}{36}$ the DFT-HSE06 functional does not provide a simple way for a Bader charge decomposition and is not used for this purpose in our study.

In order to eliminate the tunnel barrier between a contact metal and a 2D semiconductor such as $\mathrm{MoS}_{2}$, we have proposed a way to locally hole dope the channel using a stable $2 \mathrm{D}$ material. To achieve this objective without causing hybridization and the emergence of mid-gap states, we chose the highly elec- 
tronegative 2D semiconductor $\mathrm{MoO}_{3}$ or, alternatively, $\mathrm{MoO}_{2}$. Our results confirm the expectation that the band structure in the contact region closely resembles the superposition of energetically shifted bands of $\mathrm{MoS}_{2}$ and $\mathrm{MoO}_{3}$ or $\mathrm{MoO}_{2}$. We observed degenerate hole doping of the channel in contact with electronegative $\mathrm{MoO}_{3}$ and $\mathrm{MoO}_{2}$ layers. The rigid band shift in the doped contact region has an important side effect, namely a rigid shift of the channel bands in the doped with respect to the undoped channel region. This is of no consequence for the vertical tunnel barrier in the contact region, which will become negligibly small if the channel in the conducting contact region is contacted on the side opposite to molybdenum oxide by a metal. In this case, we may say that we have reached the main objective of our study, which was to optimize the metal-channel contact, using our approach.

However, another challenge has emerged that had been overlooked so far to a large degree. The rigid shift of the channel bands in the contact region causes a band offset with respect to the undoped channel region, causing the formation of an in-plane Schottky barrier. Since screening in a 2D system is much lower than in conventional 3D systems, the resulting depletion region will be larger. ${ }^{26}$ The in-layer Schottky barriers have to be considered seriously, since their effect on carrier injection may be larger than that of the vertical tunnel barriers. In that case, this lateral Schottky barrier may dominate the contact transparency.

In principle, the band offset and the associated Schottky barrier could be reduced by replacing the semiconducting channel material outside the contact region by a different, isoelectronic material. In the specific case of $p$ doped $\mathrm{MoS}_{2}$, using materials with their VBM aligned with or even higher than the shifted VBM of the channel in the contact region will eliminate such a lateral Schottky barrier and thus improve charge injection. Potential candidates for in-layer contacts with the heavily $p$ doped $\mathrm{MoS}_{2}$ segment are $\mathrm{WSe}_{2}$ and $\mathrm{MoTe}_{2}$ as the channel material. 37 Formation of such contacts by epitaxial growth of monolayer $\mathrm{WSe}_{2}$ $\mathrm{MoS}_{2}$ lateral junctions with an atomically sharp interface has recently been demonstrated. $\stackrel{38}{ }$ As an alternative, isoelectronic alloying in the channel region may be used. 39 Whether the Schottky barrier should be reduced by a lateral $2 \mathrm{D} / 2 \mathrm{D}$ junction of different materials or by isoelectronic doping, either approach will create additional interface or impurity scattering centers.

\section{Conclusions}

We have proposed an improved strategy to form low-resistance contacts to $\mathrm{MoS}_{2}$ and related semiconducting transition metal dichalcogenides by local degenerate hole doping in the contact region, where the atomically thin $2 \mathrm{D}$ channel material is in direct contact with an electronegative material such as $\mathrm{MoO}_{3}$ or $\mathrm{MoO}_{2}$. In contrast to metal contacts that are separate from the undoped 2D channel material, the homo-junction in our study is between undoped and heavily doped regions of the same $2 \mathrm{D}$ material. To check the viability of this approach, we have determined the equilibrium geometry and electronic structure of $\mathrm{MoO}_{3} / \mathrm{MoS}_{2}$ and $\mathrm{MoO}_{2} / \mathrm{MoS}_{2}$ bilayers and their monolayer components using ab initio density functional calculations. Our results indicate that, besides a rigid band shift associated with charge transfer, the presence of molybdenum oxide modifies the electronic structure of $\mathrm{MoS}_{2}$ very little, thus avoiding the formation of mid-gap states. We found that the charge transfer in the bilayer provides a sufficient degree of hole doping to $\mathrm{MoS}_{2}$ to render the contact region metallic. A highly transparent contact will thus be formed by sandwiching the semiconducting $2 \mathrm{D} \mathrm{MoS}_{2}$ channel material in-between Mo oxide and a metal.

\section{Methods}

We have studied the electronic structure, the equilibrium geometry and structural stability of $\mathrm{MoO}_{3}$ and $\mathrm{MoO}_{2}$ interacting with $\mathrm{MoS}_{2}$ using $a b$ initio density functional theory (DFT) as implemented in the VASP code. $\underline{402} \mathrm{We}$ represented these $2 \mathrm{D}$ structures by a periodic 
array of layers separated by a vacuum region in excess of $20 \AA$. We used projectoraugmented-wave (PAW) pseudopotentials, $43[44$ the Perdew-Burke-Ernzerhof $(\mathrm{PBE})^{45}$ and the optB86b-vdW $W^{46 / 47}$ exchange-correlation functionals. Since the fundamental band gap is usually underestimated in DFT-PBE calculations, we have resorted to the HSE06 ${ }^{35136}$ hybrid exchange-correlation functional, as implemented in the VASP 40 42|44 code, to get a different (possibly superior) description of the band structure. We used the default mixing parameter value $\alpha=0.25$ in these studies. The Brillouin zone of the conventional unit cell of the $2 \mathrm{D}$ structures has been sampled by a uniform $k$-point grid. ${ }^{48}$ The specific sampling we used was $15 \times 15$ for $\mathrm{MoS}_{2}, \mathrm{MoO}_{3}$ and $\mathrm{MoO}_{2}$ monolayers as well as the $\mathrm{MoO}_{2} / \mathrm{MoS}_{2}$ bilayer, $9 \times 9$ for the $\mathrm{MoO}_{3} / \mathrm{MoS}_{2}$ bilayer with the supercell A containing 14 atoms, and $2 \times 2$ for the $\mathrm{MoO}_{3} / \mathrm{MoS}_{2}$ bilayer with supercell $\mathrm{B}$ containing 156 atoms. We used $550 \mathrm{eV}$ as the electronic kinetic energy cutoff for the planewave basis and a total energy difference between subsequent self-consistency iterations below $10^{-5} \mathrm{eV} /$ atom as the criterion for reaching self-consistency. All geometries have been optimized using the conjugate-gradient method, $\underline{49}$ until none of the residual Hellmann-Feynman forces exceeded $10^{-3} \mathrm{eV} / \AA$. The maximum force criterion has been relaxed to $10^{-2} \mathrm{eV} / \AA$ in the optimization of the $\mathrm{MoO}_{3} / \mathrm{MoS}_{2}$ bilayer with the large supercell B containing 156 atoms.

\section{Supporting Information Avail- able}

The following files are available free of charge. Supercells used to represent the incommensurate $\mathrm{MoO}_{3} / \mathrm{MoS}_{2}$ bilayer. Comparison between the electronic band structure and density of states of $\mathrm{MoO}_{3} / \mathrm{MoS}_{2}$ and $\mathrm{MoO}_{2} / \mathrm{MoS}_{2}$ obtained using the DFT-HSE06 and DFT-PBE exchange-correlation functionals.

\section{Author Information}

\section{Corresponding Author}

${ }^{*}$ E-mail: tomanek@pa.msu.edu

Acknowledgement D.T. acknowledges partial support by the NSF/AFOSR EFRI 2-DARE grant number \#EFMA-1433459. Z.G. acknowledges financial support from the China Scholarship Council under Grant No. 201706260027 and the hospitality of Michigan State University. Z.Z. acknowledges partial support by NSF grant number DMR-1308436 and the WSU Presidential Research Enhancement Award. Z.G. acknowledges useful discussions with Jie Ren. Computational resources have been provided by the Michigan State University High Performance Computing Center.

\section{References}

1. Allain, A.; Kang, J.; Banerjee, K.; Kis, A. Electrical Contacts to Two-Dimensional Semiconductors. Nature Mater. 2015, 14, 1195.

2. Popov, I.; Seifert, G.; Tománek, D. Designing Electrical Contacts to $\mathrm{MoS}_{2}$ Monolayers: A Computational Study. Phys. Rev. Lett. 2012, 108, 156802.

3. Kang, J.; Liu, W.; Sarkar, D.; Jena, D.; Banerjee, K. Computational Study of Metal Contacts to Monolayer Transition-Metal Dichalcogenide Semiconductors. Phys. Rev. X 2014, 4, 031005 .

4. Lopez-Sanchez, O.; Lembke, D.; Kayci, M.; Radenovic, A.; Kis, A. Ultrasensitive Photodetectors Based on Monolayer $\mathrm{MoS}_{2} . \mathrm{Na}$ ture Nanotechnol. 2013, 8, 497.

5. Cui, X.; Lee, G.-H.; Kim, Y. D.; Arefe, G.; Huang, P. Y.; Lee, C.-H.; Chenet, D. A.; Zhang, X.; Wang, L.; Ye, F.; Pizzocchero, F.; Jessen, B. S.; Watanabe, K.; Taniguchi, T.; Muller, D. A.; Low, T.; Kim, P.; Hone, J. Multi-Terminal Transport Measurements of $\mathrm{MoS}_{2}$ Using a van der Waals Heterostructure Device Platform. Nature Nanotechnol. 2015, 10, 534. 
6. Yu, A. Y. C. Electron Tunneling and Contact Resistance of Metal-Silicon Contact Barriers. Solid-State Electron. 1970, 13, 239-247.

7. Fang, H.; Tosun, M.; Seol, G.; Chang, T. C.; Takei, K.; Guo, J.; Javey, A. Degenerate $n$-doping of Few-Layer Transition Metal Dichalcogenides by Potassium. Nano Lett. 2013, 13, 1991-1995.

8. Yang, L.; Majumdar, K.; Liu, H.; Du, Y.; Wu, H.; Hatzistergos, M.; Hung, P. Y.; Tieckelmann, R.; Tsai, W.; Hobbs, C.; Ye, P. D. Chloride Molecular Doping Technique on 2D Materials: $\mathrm{WS}_{2}$ and $\mathrm{MoS}_{2}$. Nano Lett. 2014, 14, 6275-6280.

9. Suh, J.; Park, T.-E.; Lin, D.-Y.; Fu, D.; Park, J.; Jung, H. J.; Chen, Y.; Ko, C.; Jang, C.; Sun, Y.; Sinclair, R.; Chang, J.; Tongay, S.; Wu, J. Doping Against the Native Propensity of $\mathrm{MoS}_{2}$ : Degenerate Hole Doping by Cation Substitution. Nano Lett. 2014, 14, 6976-6982.

10. Chuang, H.-J.; Chamlagain, B.; Koehler, M.; Perera, M. M.; Yan, J.; Mandrus, D.; Tománek, D.; Zhou, Z. LowResistance 2D/2D Ohmic Contacts: A Universal Approach to High-Performance $\mathrm{WSe}_{2}, \mathrm{MoS}_{2}$, and $\mathrm{MoSe}_{2}$ Transistors. Nano Lett. 2016, 16, 1896-1902.

11. Fang, H.; Chuang, S.; Chang, T. C.; Takei, K.; Takahashi, T.; Javey, A. HighPerformance Single Layered $\mathrm{WSe}_{2} p$-FETs with Chemically Doped Contacts. Nano Lett. 2012, 12, 3788-3792.

12. Kiriya, D.; Tosun, M.; Zhao, P.; Kang, J. S.; Javey, A. Air-Stable Surface Charge Transfer Doping of $\mathrm{MoS}_{2}$ by Benzyl Viologen. J. Am. Chem. Soc. 2014, 136, 7853-7856.

13. Cai, L.; McClellan, C. J.; Koh, A. L.; Li, H.; Yalon, E.; Pop, E.; Zheng, X. Rapid Flame Synthesis of Atomically Thin $\mathrm{MoO}_{3}$ down to Monolayer Thickness for Effective Hole Doping of $\mathrm{WSe}_{2}$. Nano Lett. 2017, 17, 3854-3861.
14. Tománek, D. Interfacing Graphene and Related 2D Materials with the 3D World. J. Phys.: Condens. Matter 2015, 27, 133203.

15. Chen, W.; Santos, E. J.; Zhu, W.; Kaxiras, E.; Zhang, Z. Tuning the Electronic and Chemical Properties of Monolayer $\mathrm{MoS}_{2}$ Adsorbed on Transition Metal Substrates. Nano Lett. 2013, 13, 509-514.

16. Gong, C.; Colombo, L.; Wallace, R. M.; Cho, K. The Unusual Mechanism of Partial Fermi Level Pinning at Metal-MoS 2 Interfaces. Nano Lett. 2014, 14, 1714-1720.

17. Movva, H. C.; Rai, A.; Kang, S.; Kim, K.; Fallahazad, B.; Taniguchi, T.; Watanabe, K.; Tutuc, E.; Banerjee, S. K. HighMobility Holes in Dual-Gated $\mathrm{WSe}_{2}$ Fieldeffect Transistors. ACS Nano 2015, 9, 10402-10410.

18. Chen, J.-R.; Odenthal, P. M.; Swartz, A. G.; Floyd, G. C.; Wen, H.; Luo, K. Y.; Kawakami, R. K. Control of Schottky Barriers in Single Layer $\mathrm{MoS}_{2}$ Transistors with Ferromagnetic Contacts. Nano Lett. 2013, 13, 3106-3110.

19. Wang, J.; Yao, Q.; Huang, C.-W.; Zou, X.; Liao, L.; Chen, S.; Fan, Z.; Zhang, K.; Wu, W.; Xiao, X.; Jiang, C.; Wu, W.-W. High Mobility $\mathrm{MoS}_{2}$ Transistor with Low Schottky Barrier Contact by Using Atomic Thick $h-\mathrm{BN}$ as a Tunneling Layer. Adv. Mater. 2016, 28, 8302-8308.

20. Cui, X.; Shih, E.-M.; Jauregui, L. A.; Chae, S. H.; Kim, Y. D.; Li, B.; Seo, D.; Pistunova, K.; Yin, J.; Park, J.-H.; Choi, H.-J.; Lee, Y. H.; Watanabe, K.; Taniguchi, T.; Kim, P.; Dean, C. R.; Hone, J. C. Low-Temperature Ohmic Contact to Monolayer $\mathrm{MoS}_{2}$ by van der Waals Bonded Co/h-BN Electrodes. Nano Lett. 2017, 17, 4781-4786.

21. Guan, J.; Chuang, H.-J.; Zhou, Z.; Tománek, D. Optimizing Charge Injection across Transition Metal Dichalcogenide Heterojunctions: Theory and Experiment. ACS Nano 2017, 11, 3904-3910. 
22. Kappera, R.; Voiry, D.; Yalcin, S. E.; Branch, B.; Gupta, G.; Mohite, A. D.; Chhowalla, M. Phase-Engineered Lowresistance Contacts for Ultrathin $\mathrm{MoS}_{2}$ Transistors. Nature Mater. 2014, 13, 1128.

23. Cho, S.; Kim, S.; Kim, J. H.; Zhao, J.; Seok, J.; Keum, D. H.; Baik, J.; Choe, D.H.; Chang, K. J.; Suenaga, K.; Kim, S. W.; Lee, Y. H.; Yang, H. Phase Patterning for Ohmic Homojunction Contact in $\mathrm{MoTe}_{2}$. Science 2015, 349, 625-628.

24. Yang, H.; Kim, S. W.; Chhowalla, M.; Lee, Y. H. Structural and Quantum-State Phase Transitions in van der Waals Layered Materials. Nature Phys. 2017, 13, 931.

25. Heinze, S.; Tersoff, J.; Martel, R.; Derycke, V.; Appenzeller, J.; Avouris, P. Carbon Nanotubes as Schottky Barrier Transistors. Phys. Rev. Lett. 2002, 89, 106801.

26. Yi, Y.; Wu, C.; Liu, H.; Zeng, J.; He, H.; Wang, J. A Study of Lateral Schottky Contacts in $\mathrm{WSe}_{2}$ and $\mathrm{MoS}_{2}$ Field Effect Transistors Using Scanning Photocurrent Microscopy. Nanoscale 2015, 7, 15711-15718.

27. Farmanbar, M.; Brocks, G. Ohmic Contacts to 2D Semiconductors through van der Waals Bonding. Adv. Electr. Mater. 2016, 2, 1500405.

28. Greiner, M. T.; Helander, M. G.; Tang, W.M.; Wang, Z.-B.; Qiu, J.; Lu, Z.-H. Universal Energy-Level Alignment of Molecules on Metal Oxides. Nature Mater. 2012, 11, 76.

29. Xie, L.; Wang, X.; Mao, H.; Wang, R.; Ding, M.; Wang, Y.; Özyilmaz, B.; Ping Loh, K.; Wee, A. T.; Ariando,; Chen, W. Electrical Measurement of Nondestructively $p$-Type Doped Graphene Using Molybdenum Trioxide. Appl. Phys. Lett. 2011, 99, 012112.

30. Russell, S. A.; Cao, L.; Qi, D.; Tallaire, A.; Crawford, K. G.; Wee, A. T.; Moran, D. A. Surface Transfer Doping of Diamond by $\mathrm{MoO}_{3}$ : A Combined Spectroscopic and
Hall Measurement Study. Appl. Phys. Lett. 2013, 103, 202112.

31. Chuang, S.; Battaglia, C.; Azcatl, A.; McDonnell, S.; Kang, J. S.; Yin, X.; Tosun, M.; Kapadia, R.; Fang, H.; Wallace, R. M.; Javey, A. MoS 2 p-Type Transistors and Diodes Enabled by High Work Function $\mathrm{MoO}_{x}$ Contacts. Nano Lett. 2014, 14, 1337-1342.

32. Xia, F.; Shao, Z.; He, Y.; Wang, R.; Wu, X.; Jiang, T.; Duhm, S.; Zhao, J.; Lee, S.T.; Jie, J. Surface Charge Transfer Doping via Transition Metal Oxides for Efficient $p$-Type Doping of II-VI Nanostructures. ACS Nano 2016, 10, 10283-10293.

33. Sivacarendran, B.; Junkai, D.; Zhen, O. J.; Sumeet, W.; James, S.; Jianshi, T.; L., W. K.; R., F. M.; Salvy, R.; Serge, Z.; S., S. M.; Nikhil, M.; Sharath, S.; Madhu, B.; Zadeh Kourosh, K. Enhanced Charge Carrier Mobility in TwoDimensional High Dielectric Molybdenum Oxide. Adv. Mater. 2013, 25, 109-114.

34. Ataca, C.; Şahin, H.; Ciraci, S. Stable, Single-layer $\mathrm{MX}_{2}$ Transition-Metal Oxides and Dichalcogenides in a Honeycomb-Like Structure. J. Phys. Chem. C 2012, 116, 8983-8999.

35. Heyd, J.; Scuseria, G. E.; Ernzerhof, M. Hybrid Functionals Based on a Screened Coulomb Potential. J. Chem. Phys. 2003, 118, 8207-8215.

36. Krukau, A. V.; Vydrov, O. A.; Izmaylov, A. F.; Scuseria, G. E. Influence of the Exchange Screening Parameter on the Performance of Screened Hybrid Functionals. J. Chem. Phys. 2006, 125, 224106.

37. Gong, C.; Zhang, H.; Wang, W.; Colombo, L.; Wallace, R. M.; Cho, K. Band Alignment of Two-Dimensional Transition Metal Dichalcogenides: Application in Tunnel Field Effect Transistors. Appl. Phys. Lett. 2013, 103, 053513. 
38. Li, M.-Y.; Shi, Y.; Cheng, C.-C.; Lu, L.S.; Lin, Y.-C.; Tang, H.-L.; Tsai, M.L.; Chu, C.-W.; Wei, K.-H.; He, J.-H.; Chang, W.-H.; Suenaga, K.; Li, L.-J. Epitaxial Growth of a Monolayer $\mathrm{WSe}_{2}-\mathrm{MoS}_{2}$ Lateral $p-n$ Junction with an Atomically Sharp Interface. Science 2015, 349, 524528.

39. Duan, X.; Wang, C.; Fan, Z.; Hao, G.; Kou, L.; Halim, U.; Li, H.; Wu, X.; Wang, Y.; Jiang, J.; Pan, A.; Huang, Y.; $\mathrm{Yu}, \mathrm{R}$.; Duan, X. Synthesis of $\mathrm{WS}_{2 x} \mathrm{Se}_{2-2 x}$ Alloy Nanosheets with CompositionTunable Electronic Properties. Nano Lett. 2015, 16, 264-269.

40. Kresse, G.; Furthmüller, J. Efficient Iterative Schemes for Ab Initio Total-Energy Calculations using a Plane-Wave Basis Set. Phys. Rev. B 1996, 54, 11169-11186.

41. Kresse, G.; Furthmüller, J. Efficiency of $A b$ Initio Total Energy Calculations for Metals and Semiconductors Using a Plane-Wave Basis Set. Computat. Mater. Sci. 1996, 6, $15-50$.

42. Kresse, G.; Hafner, J. Ab Initio MolecularDynamics Simulation of the Liquid-MetalAmorphous-Semiconductor Transition in Germanium. Phys. Rev. B 1994, 49, 14251-14269.

43. Blöchl, P. E. Projector Augmented-Wave Method. Phys. Rev. B 1994, 50, 1795317979 .

44. Kresse, G.; Joubert, D. From Ultrasoft Pseudopotentials to the Projector Augmented-Wave Method. Phys. Rev. B 1999, 59, 1758-1775.

45. Perdew, J. P.; Burke, K.; Ernzerhof, M. Generalized Gradient Approximation Made Simple. Phys. Rev. Lett. 1996, 77, 38653868.

46. Klimeš, J.; Bowler, D. R.; Michaelides, A. Chemical Accuracy for the van der Waals Density Functional. J. Phys. Condens. Matter 2010, 22, 022201.
47. Klimeš, J.; Bowler, D. R.; Michaelides, A. Van der Waals Density Functionals Applied to Solids. Phys. Rev. B 2011, 83, 195131.

48. Monkhorst, H. J.; Pack, J. D. Special Points for Brillouin-Zone Integrations. Phys. Rev. B: Condens. Matter Mater. Phys. 1976, 13, 5188-5192.

49. Hestenes, M. R.; Stiefel, E. Methods of Conjugate Gradients for Solving Linear Systems. J. Res. Natl. Bur. Stand. 1952, 49, 409-436. 